- the change in the structure of the production factors and their combinations often makes impossible the price compensations at the level of their income.

-- the increase of the size of the pig enterprise results in a disproportion between one of the enterprises and the other farm productions a price compensation being consequently impossible. However, the enterprises do not passively support these perturbations, on the contrary, they try to establish a relative stability. This policy of stabilization has a price. It is on the basis of such an analysis that certain developments of the production structures can be revealed;

- the cost of stabilization tends to reduce and even to annihilate the gain of productivity which may be realized by the modern production units. This fact explains the slowness of the growth of the modern sector and the survival of the units of traditional and relative small-units ;

- this fact also partly explains the reasons why a certain number of agents of the pig branch undertake expensive operations of capital integration being very often unprofitable.

- integration of breeding by the pig feeders ;

- integration or quasi integration of the pig production by the input supplying industries and the processing industries.

\title{
LES PERSPECTIVES DANS L'INDUSTRIE DE TRANSFORMATION DE LA VIANDE PORCINE
}

\author{
J. HOSSENLOPP \\ Laboratoire de Recherches sur l'Économie des Industries alimentaires, \\ C. E. R. D. I. A., \\ 91 - Massy \\ Institut national de la Recherche agronomique
}

\section{RÉSUMÉ}

La nécessité d'une réorganisation du secteur de transformation de la viande porcine a été ressentie depuis plusieurs années. Deux éléments favorables jouent dans ce sens; d'une part la modification de l'appareil de distribution des produits alimentaires, et d'autre part les changements qualitatifs de la consommation. Une analyse de la situation montre que les changements déjà opérés ne sont en rien comparables à ceux qui vont se produire dans les cinq prochaines années Les retards importants accumulés face aux concurrents et les progrès à accomplir sont des éléments convaincants dans l'appréciation des mutations à venir dans nos industries de transformation. L'évolution peut être réalisée grâce à des facteurs internes, tels que le regroupement et la coordination entre des entreprises existantes, pouvant faire contrepoids aux formes de distributions modernes. Certains ateliers pourront être ainsi spécialisés sur certains produits. Mais on peut 
également penser qu'il restera quelques entreprises non spécialisées à vocation régionale. L'évolution peut être également induite par les agents extérieurs que sont les pressions de l'État, l'intervention d'organismes financiers, la concurrence étrangère sur les produits transformés, la prise de participation ou de contrôle par des firmes extérieures au secteur de la transformation, ou par des firmes multinationales puissantes. Ce dernier processus est déjà engagé.

\title{
SUMMARY
}

\section{PERSPECTIVES FOR THE PORK TRANSFORMATION INDUSTRY}

It has been apparent for several years that a re-organization of the pork transformation industry is necessary. Two favorable elements play a role in this : modification of the system of food products distribution and qualitative consumption changes. When the situation is analyzed, it appears that the changes which have already come about are nothing compared to those which occur in the next 5 years. Great backwardness as regards competitors and progress yet to be accomplished, are convincing elements in appreciating modifications to come in our transformation industries. Progress will be realized by internal factors such as re-grouping and coordination between existing enterprises. These will carry weight against modern forms of distribution. Some enterprises may be specialized in certain products. However, there will probably always be some unspecialized ones of regional vocation. Progress may also result from exterior factors such as government pressure, pressure of financial organizations, foreign competition in transformed products, and participation or control by companies outside the transformation sector, or by powerful multinational firms. The latter process has already started.

\section{LE RÔLE DES TRANSFORMATIONS DE L'AVAL dans L'Évolution de L'INDUSTRie de la Viande de porc}

\author{
H. DULEY
}

\author{
Laboratoire de Recherches sur l'Économie des Industries alimentaires, \\ C. E. R. D. I. A., \\ 91 - Massy \\ Institut national de la Recherche agronomique
}

\section{RÉSUMÉ}

Les changements de l'aval des industries de transformation du porc ont une importance fondamentale pour l'avenir de l'ensemble de la filière porc (production et industries de transformation). Nous pouvons les observer aux deux niveaux de la consommation et de la distribution. 